\title{
Mesalazine-induced bullous fixed drug eruption
}

\section{Names of authors:}

Andac $\underline{\text { Salman }},{ }^{1}$ Dilek Seckin Gencosmanoglu,${ }^{1}$ Yesim Ozen Alahdab, ${ }^{2}$ Ana Maria Giménez$\underline{\operatorname{Arnau}}^{3}$

\section{Name of departments:}

${ }^{1}$ Department of Dermatology, Marmara University School of Medicine, 34899, Istanbul, Turkey

${ }^{2}$ Department of Gastroenterology, Marmara University School of Medicine, 34899, Istanbul, Turkey

${ }^{3}$ Department of Dermatology, Hospital del Mar, Universitat Autònoma de Barcelona, 08004, Barcelona, Spain

Corresponding author: Andac Salman

Address: Marmara Universitesi Pendik Egitim ve Arastirma Hastanesi

Dermatoloji Anabilim Dali Fevzi Cakmak Mah, Mimar Sinan Cad. No:41

34899 Pendik, Istanbul, Turkey

Tel: +902166570606-extension: 3240/4219

E-mail: asalmanitf@gmail.com, andac.salman@marmara.edu.tr

Sources of funding: None.

Conflict of interest: None declared.

Author contributions: Design (AS, DSG, YOA, AGA); acqusition and interpretation of data (AS, DSG, AGA); drafting and critical revision (AS, DSG, YOA, AGA); final approval (AS, DSG, YOA, AGA).

Running head: Fixed drug eruption due to mesalazine

Keywords: 5-aminosalicylic acid, case report, fixed drug eruption, mesalazine. 


\section{Introduction}

Mesalazine, also known as 5-aminosalicylic acid (5-ASA) or mesalamine, is an antiinflammatory drug which is used in the treatment of ulcerative colitis and Crohn's disease (1). Mesalazine induced cutaneous adverse reactions such as non-specific rash, acute generalized exanthematous pustulosis (AGEP) and toxic epidermal necrolysis (TEN) have been reported (2-5). Herein, we report a patient with bullous fixed drug eruption (bFDE) due to mesalazine, which has not been reported previously, to our knowledge.

\section{Case Report}

A 35-year-old man with development of vesicular and bullous lesions always, at the same site of his feet, following mesalazine (Asacol) intake prescribed for ulcerative colitis is described (Fig. 1a-e). He developed at least two episodes of the diagnosed bFDE, after taking oral and suppository forms of mesalazine. He did not suffer any previous similar complaints with other concomitantly used pharmaceutical products. His medical history was insignificant except for ulcerative colitis.

Dermatological examination revealed brown-violaceous, slightly crusted, ovale plaques located symmetrically on the plantar aspects of his feet (Fig. 1c, d). Patient's own clinical photographs showed bullous lesions always on the same sites. (Fig. 1a, b). A presumptive diagnosis of bFDE was made based on the clinical findings and temporal relationship between mesalazine intake and development of lesions.

Patch testing with Asacol (mesalazine) tablets diluted in petrolatum (10\%, 1\% and $0.1 \%$ ) was performed on the lesional skin and on the upper back of the patient to confirm the diagnosis, only six weeks after the clinical resolution of the lesions. Readings were recorded on day (D) 2 and D5, according to ESCD guidelines (6). On D2 and D5 there was a weak positive reaction on the upper back and a strong positive reaction over the lesional skin with all the concentrations tested, confirming the diagnosis of bFDE due to mesalazine (Fig. 2a-e). 


\section{Discussion}

Aminosalicylates (5-ASA), including mesalazine and sulfasalazine, are among the first line medical therapies for inflammatory bowel diseases $(1,7)$. Although sulfasalazine is metabolized into its two components, sulfapyridine and mesalazine, in the colon, mesalazine and sulfasalazine have been reported to display a different spectrum of adverse effects (8). In addition to being one of the most renowned causes of drug reaction with eosinophilia and systemic symptoms (DRESS) (9), sulfasalazine has also been associated with fixed drug eruption (FDE) (10,11). Sulfasalazine-induced FDE involved oral and genital mucosa, and presented with hyperpigmented macules and erosions in both cases $(10,11)$. Sulfasalazine as offending drug was confirmed by oral provocation tests in one patient (10) and patch testing in another other patient (11). However, mesalazine-induced FDE has not been reported to date.

Our report underscores how useful patch testing is confirming which is the culprit drug involved in fixed drug eruption. Consequently, a more proper guidance on treatment decisions can be addressed in this patient. 


\section{References}

1. Mowat C, Cole A, Windsor A, et al. Guidelines for the management of inflammatory bowel disease in adults. Gut 2011: 60: 571-607.

2. Fukunaga K, Ohda Y, Inoue T, et al. Toxic epidermal necrosis associated with mesalamine in a patient with ulcerative colitis. Inflamm Bowel Dis 2007: 13: 10551056.

3. Rocci E, Park K, Hutchens K, Winterfield L. First report of mesalamine (5aminosalicylic acid) as the causative agent in a case of acute generalized exanthamous pustulosis. Dermatol Online J 2017: 23.

4. Gibbon KL, Bewley AP, Thomas K. Mesalazine-induced pustular drug eruption. J Am Acad Dermatol 2001: 45: s220-221.

5. Brimblecombe R. Mesalazine: a global safety evaluation. Scand J Gastroenterol Suppl 1990: 172: 66.

6. Johansen JD, Aalto-Korge K, Agner T, et al. European Society of Contact Dermatitis guideline for diagnostic patch testing - recommendations on best practice. Contact Dermatitis 2015: 73: 195-221.

7. Cheifetz AS. Management of active Crohn disease. JAMA 2013: 309: 2150-2158.

8. Ransford RA, Langman MJ. Sulphasalazine and mesalazine: serious adverse reactions re-evaluated on the basis of suspected adverse reaction reports to the Committee on Safety of Medicines. Gut 2002: 51: 536-539.

9. Cacoub P, Musette P, Descamps V, et al. The DRESS syndrome: a literature review. Am J Med 2011: 124: 588-597.

10. Kanwar AJ, Singh M, Yunus M, Belhaj MS. Fixed eruption to sulphasalazine. Dermatologica 1987: 174: 104. 
11. Kawada A, Kobayashi T, Noguchi H, et al. Fixed drug eruption induced by sulfasalazine. Contact Dermatitis 1996: 34: 155-156. 


\section{Figure legends}

Figure 1. Patient's self-taken clinical photographs showing symmetric bullous lesions on the plantar aspect of the feet $(a, b)$. Progression of the lesions into hyperpigmented, scaly plaques (c, d) and residual post-inflammatory hyperpigmentation (e).

Figure 2. Patch test readings. Weak positive reaction on D2 and D5 on the upper back (a, b). Strong positive reaction on the lesional skin on D2 and D5 (c-e). 